\title{
TNF- $\alpha$-mediated inflammation in cerebral aneurysms: A potential link to growth and rupture
}

\author{
Thottala Jayaraman ${ }^{1,2}$ \\ Andrew Paget ${ }^{\prime}$ \\ Yang Sam Shin' \\ Xiaguai Li' \\ Jillian Mayer' \\ Hina W Chaudhry ${ }^{2}$ \\ Yasunari $\mathrm{Niimi}^{3}$ \\ Michael Silane ${ }^{4}$ \\ Alejandro Berenstein ${ }^{3}$ \\ 'Department of Neurosurgery, \\ St. Luke's Roosevelt Hospital Center, \\ New York, NY, USA; ${ }^{2}$ Department \\ of Medicine, College of Physicians \\ and Surgeons, Columbia University, \\ New York, NY, USA; ${ }^{3}$ Endovascular \\ Surgery, St. Luke's Roosevelt Hospital \\ Center, New York; and ${ }^{4}$ Division \\ of Vascular Surgery, Beth Israel \\ Medical Center, New York, NY, USA
}

Correspondence: Thottala Jayaraman Vascular Biology Laboratory, St. Luke's Roosevelt Hospital Center, III I

Amsterdam Avenue, New York, NY 10025 USA

Tel +I 2125232220

Fax + I 212523 1694

Email tj56@columbia.edu

\begin{abstract}
Intracranial aneurysm (IA) rupture is one of the leading causes of stroke in the United States and remains a major health concern today. Most aneurysms are asymptomatic with a minor percentage of rupture annually. Regardless, IA rupture has a devastatingly high mortality rate and does not have specific drugs that stabilize or prevent aneurysm rupture, though other preventive therapeutic options such as clipping and coiling of incidental aneurysms are available to clinicians. The lack of specific drugs to limit aneurysm growth and rupture is, in part, attributed to the limited knowledge on the biology of IA growth and rupture. Though inflammatory macrophages and lymphocytes infiltrate the aneurysm wall, a link between their presence and aneurysm growth with subsequent rupture is not completely understood. Given our published results that demonstrate that the pro-inflammatory cytokine, tumor necrosis factor-alpha (TNF- $\alpha$ ), is highly expressed in human ruptured aneurysms, we hypothesize that pro-inflammatory cell types are the prime source of TNF- $\alpha$ that initiate damage to endothelium, smooth muscle cells (SMC) and internal elastic lamina (IEL). To gain insights into TNF- $\alpha$ expression in the aneurysm wall, we have examined the potential regulators of TNF- $\alpha$ and report that higher TNF- $\alpha$ expression correlates with increased expression of intracellular calcium release channels that regulate intracellular calcium $\left(\mathrm{Ca}^{2+}\right)_{\mathrm{i}}$ and Toll like receptors (TLR) that mediate innate immunity. Moreover, the reduction of tissue inhibitor of metalloproteinase-1 (TIMP-1) expression provides insights on why higher matrix metalloproteinase (MMP) activity is noted in ruptured IA. Because TNF- $\alpha$ is known to amplify several signaling pathways leading to inflammation, apoptosis and tissue degradation, we will review the potential role of TNF- $\alpha$ in IA formation, growth and rupture. Neutralizing TNF- $\alpha$ action in the aneurysm wall may have a beneficial effect in preventing aneurysm growth by reducing inflammation and arterial remodeling.

Keywords: innate immunity, TNF- $\alpha$, inflammation, intracranial aneurysm growth
\end{abstract}

\section{Introduction}

Intracranial aneurysm (IA) is prevalent among both elderly men and women. In the United States, unruptured IA is present in approximately $4 \%-5 \%$ of the general population. Many IA are clinically silent until they rupture with devastating clinical consequences. Although only $1 \%-2 \%$ of IA ruptures annually, the neurological damage associated with rupture is often irreversible (Juvela et al 2000; Peters et al 2001; Ruigrok et al 2005; Nahed et al 2007). Approximately $1 / 3$ of ruptured IA is fatal, with $1 / 3$ of patients suffering notable neurological damage, and the remaining $1 / 3$ of patients are neurologically normal following subarachnoid hemorrhage (SAH). Despite their clinical importance, the pathogenesis of IA remains obscure.

Although the exact etiology of IA disease is not known, there are many risk factors that are associated with the weakening of cerebral arteries (Ostergaard 1989; Juvela 2002; Hashimoto et al 2006). They include hypertension, hemodynamic stress, atherosclerosis, smoking, aging, and genetic abnormalities such as Marfan syndrome, pseudoxanthoma elasticum, Ehlers-Danlos syndrome, fibromuscular dysplasia, and polycystic kidney disease. 
Therefore, it was suggested that aneurysms, namely berry type, infectious, dissecting, and giant partially thrombosed, are different with respect to their behavior, topography, localization, and association with certain genetic or familial diseases and represent a wide variety of diseases triggered by hemodynamic and nonhemodynamic factors. The berry type is not regarded as the same disease as the giant chronic dissecting aneurysms because the latter demonstrate a multifocal character suggesting nonhemodynamic triggering factors. In addition, giant aneurysms behave differently over time, with about 50\% thrombosing, whereas only $2 \%$ of berry aneurysms show complete thrombosis. In this study, Zhao and his colleagues (2004) have noted that the weakening of aneurysmal artery does not occur from the inside of the vessel, rather it is caused from the outside by abluminal factors via leukotrienes (LT) release by 5-LO pathway that initiates adventitial inflammation by weakening the media and dilating the vessel conducive for aneurysm formation.

While this and other studies reinforce that inflammation plays a role in aneurysm pathogenesis, a common pathogenic mechanism associated with these risk factors has not yet been identified. Given several risk factors are associated with aneurysm growth, it is not unreasonable to expect the involvement of multiple signaling molecules or pathways. Recently, we have reported that the pro-inflammatory cytokine, tumor necrosis factor-alpha (TNF- $\alpha$ ), is highly expressed in cerebral aneurysm walls (Jayaraman et al 2005) and propose that its expression and activation by these risk factors can be linked to the onset of IA through a remarkable series of correlations. In this review, we provide an overview of its potential role in aneurysm formation with molecular insights gained from tumor necrosis factor receptor (TNFR) signaling and TNF- $\alpha$-dependent cellular mechanisms.

\section{Evidence for the presence of inflammation in cerebral aneurysms}

Inflammation is a vital process that plays a key role in health and disease (Henry and Chen 1993; Slifka and Whitton 2000; Hotamisligil 2006). It is initiated by a complex series of events that lead to changes in vascular wall integrity, and emerges as a shared pathological mechanism in a variety of vascular diseases, including atherosclerosis, abdominal aortic aneurysm, and arteritis (Henry and Chen 1993; Jang et al 1993). Although inflammation has been suggested to be an underlying cause of cerebral aneurysms (Chyatte et al 1999), it was largely ignored until recent findings demonstrated the presence of inflammatory cells (macrophages,
T- and B-lymphocytes) in the walls of human IA (Chyatte et al 1999; Kataoka et al 1999) with increased levels of matrix metalloproteinases and other proteolytic enzymes in the serum of aneurysm patients (Toder et al 1998) and polymorphisms (Zhang et al 2001; Krex et al 2003). Complement, an important part of the innate and adaptive immune system, was found in almost all aneurysms studied. Both $\mathrm{C} 3$, the most abundant complement protein in the blood, and C9, the terminal protein of the complement cascade, were found within cerebral aneurysms, and together act as a marker for complement activation (Chyatte et al 1999). The fact that half of all unruptured aneurysms (10/20) and all of the ruptured aneurysms (40/40) are infiltrated with inflammatory leukocytes suggests that inflammatory cells and their mediators contribute to aneurysm growth and rupture (Kataoka et al 1999). However, it is not known if different subsets of inflammatory leukocytes dominate in ruptured as compared with nonruptured aneurysms.

\section{Pro-inflammatory protein mediators}

Inflammation in the aneurysm wall is likely to be controlled by the relative balance of pro- and anti-inflammatory cytokines (Slifka and Whitton 2000; Benelli et al 2006). Some of the prominent pro-inflammatory cytokines include interleukin $1 \beta$ (IL-1 $\beta$ ), IL-6, IL-8, IL-18, interferon-gamma (IFN- $\gamma$ ), and TNF- $\alpha$, predominantly secreted by macrophages and to a lesser extent by lymphocytes (Xing et al 1998; Aggarwal and Takada 2005). These cytokines may increase systemic inflammatory processes by upregulating acute phase protein synthesis and promoting permeability across blood-brain barrier (BBB) into the aneurysm wall.

Several groups have analyzed cytokine mRNA and protein expression in IA and it is now apparent that many cytokines are expressed abundant in IA including IL-1 $\beta$, IL-6, TNF$\alpha$, and chemokines, IL- 8 , and monocyte chemo-attractant protein-1 (MCP-1) (Cao et al 2002; Jayaraman et al 2005; Morgan et al 2006; Moriwaki et al 2006). While these studies indicate that cytokine expression in IA walls may be prolonged, it is not known whether they are a cause or an effect of aneurysm formation. Moreover, a plethora of cytokines detected in IA would suggest the existence of a hierarchy in terms of importance in the pathogenesis of IA. TNF- $\alpha$ 's ability to regulate other pro-inflammatory cytokines is demonstrated by TNF- $\alpha$ neutralization studies in rheumatoid arthritis (RA) with markedly reduced production of IL-1, IL-6, and IL-8 (Maini et al 1995). These results imply that in inflammatory disease states, pro-inflammatory cytokines are linked 
in a sequence resembling an electrical circuit in series, with TNF- $\alpha$ at the apex of the cascade in IA inducing production of IL-1, and both TNF- $\alpha$ and IL-1 inducing downstream cytokines such as IL-6 and IL-8. The lack of IL-10 in human ruptured aneurysms suggests that anti-inflammatory cytokines are not either activated or suppressed in the aneurysm wall. Absence of IL-10 generation could be due to either T helper2 (Th2) cells being absent or suppressed. Our data points to the latter, as our unpublished results show that Th2 cell types are present. In addition to pro-inflammatory cytokines, other mediators may also be involved. However, it is not known if their activation is the cause or effect of inflammation mediated by pro-inflammatory cytokines (Morgan et al 2005).

\section{Pro-inflammatory lipid mediators}

In addition to protein pro-inflammatory mediators such as TNF- $\alpha$, lipid mediators can also cause inflammation in vascular walls. 5-lipooxygenase (5-LO) has recently attracted attention because of their involvement in synthesis of leukotriene (LT), a group of pro-inflammatory lipid mediators derived from arachidonic acid. Both experimental and clinical studies implicate 5-LO pathway in the pathophysiology of asthma and atherosclerosis. The expressions of 5-LO in mammals are tightly regulated and are modulated in vitro by calcium $\left(\mathrm{Ca}^{2+}\right)$, adenosine triphosphate (ATP), phosphatidylcholine, and lipid hydroperoxides. $\mathrm{Ca}^{2+}$ ions $(0.5 \mu \mathrm{M}$ concentrations or higher $)$, but not $\mathrm{Mg}^{2+}$ or $\mathrm{Mn}^{2+}$, activate 5-LO by dimerizing the inactive 5-LO into an active form (180 KD) (Parker and Aykent 1982). Interestingly, LO-5 pathway is also known to induce proinflammatory cytokines, including TNF- $\alpha$ generation, via LT production. For instance, LTB4 of synovial membrane cells in rheumatoid arthritis induce TNF- $\alpha$ and IL- $1 \beta$ expression at mRNA level (Chen and Lv 2006) and 5-LO inhibitors, VZ-65 and AA-861, significantly inhibit TNF- $\alpha$ production, whether administered simultaneously with lipopolysaccharide (LPS) or $30 \mathrm{~min}$ after LPS treatment. While these studies suggest that 5 -LO can induce TNF- $\alpha$ expression, whether TNF- $\alpha$, as a downstream signal, could also be involved in giant aneurysms pathogenesis is yet to be determined.

Though we have not determined 5-LO in our study, increased IP3R expression suggests that several $\mathrm{Ca}^{2+}$ dependent pro-signaling molecules, including 5-LO, might be activated in aneurysm wall. Our findings thus provide a novel mechanism by which different signaling pathways are activated in aneurysm wall and a new strategy for preventing IA growth and rupture.

\section{TNF- $\alpha$ links risk factors with aneurysm development}

Multiple systemic risk factors, including hypertension, hemodynamic stress, age ( $>50$ years), atherosclerosis, smoking, alcohol consumption, and gender differences (females have higher cerebral aneurysm rate as compared with men with $\sim 1.6$ to 1 ratio), are involved in the pathogenesis of IA. However, it has not been possible to associate these risk factors with a common pathogenic mechanism. We have shown that TNF- $\alpha$ is significantly expressed in ruptured cerebral aneurysm walls, whose expression/activity can also be linked with cerebral aneurysms growth. These include observations that 1) TNF- $\alpha$ generation occurs in response to each of these diverse risk factors associated with cerebral aneurysm growth (Table 1); 2) atherosclerotic lesions form

Table I Tumor necrosis factor-alpha (TNF- $\alpha$ ) generation is a common signal associated with intracranial aneurysm risk factors. The studies show that TNF- $\alpha$ induction is associated with each of the known aneurysm risk factors

\begin{tabular}{|c|c|c|}
\hline Risk factors & TNF- $\alpha$ induction & References \\
\hline Hemodynamic flow & Yes & $\begin{array}{l}\text { Gonzalez et al 1992; Kapadia et al 2000; Hoi et al } \\
2004 \text {; Jou et al } 2005\end{array}$ \\
\hline Hypertension & Yes & Munoz et al 1999 \\
\hline Aging & $\begin{array}{l}\text { Yes, intracellular expression of TNF- } \alpha \\
\text { increases progressively with age }\end{array}$ & $\begin{array}{l}\text { Paolisso et al 1998; Kirwan et al 200 I; Hartel } \\
\text { et al } 2005\end{array}$ \\
\hline Gender & Yes, estrogen inhibits TNF- $\alpha$ activity & $\begin{array}{l}\text { Puder et al 200I; Schrman et al 200I; Huang et al } \\
\text { 2005; Ferreri } 2007\end{array}$ \\
\hline Smoking & $\begin{array}{l}\text { Yes, smoking increases residual TNF- } \alpha \\
\text { activity in vessels }\end{array}$ & Anto et al 2002; Sun et al 2007; Wright et al 2007 \\
\hline Alcohol & Yes & $\begin{array}{l}\text { Gonazalez-QA 2004; Luedemann et al 2005; } \\
\text { Lanzke et al } 2007\end{array}$ \\
\hline Genetic & Yes & $\begin{array}{l}\text { Zhai et al I997; Gregersen et al 2000; Ruetzler } \\
\text { et al 200I; Lambertsen et al } 2002\end{array}$ \\
\hline
\end{tabular}

Abbreviation: TNF- $\alpha$, tumor necrosis factor-alpha 
at distinct regions of the arterial tree, especially at or near branch points or major vascular curvatures where TNF- $\alpha$ is selectively expressed; and 3) inflammatory macrophages and lymphocytes are present in the aneurysm wall (Chyatte et al 1999). Although these correlations are provocative, there is no direct evidence demonstrating that TNF- $\alpha$ activation is necessary or sufficient for aneurysm formation. Given TNF- $\alpha$ alters BBB functions permitting inflammatory cells across the BBB to the vascular injury site, we propose that TNF- $\alpha$ generation by these risk factors is an important early signal that initiates the groundwork for cerebral aneurysm development and growth via its signaling activation.

In addition to activating specific signaling events, TNF- $\alpha$ can also have indirect effects on the aneurysm wall through activation of other signaling molecules such as tissue factor (TF), adhesion molecules for leukocytes, release of IL-1, nitric oxide, factor VIII/von Willebrand factor, platelet activating factor, endothelin, which will have profound effect on the arterial wall (Tracy et al 1993). The effect of TNF- $\alpha$ on intracranial vessel has been studied in stroke models. For instance, intracranial blood vessel activation by either intracisternally administered LPS or chronic activation of BCG-primed monocytes/macrophages renders rats sensitive to hemorrhage (Sirén et al 2001). The fact that administration of a recombinant type 1 soluble TNF receptor (TNF binding protein) prevents these strokes suggests TNF- $\alpha$ as a necessary casual factor. Moreover, the gene expression pattern by TNF- $\alpha$ and LPS appears to be very similar (Magder et al 2006). The role of TNF- $\alpha$ in stroke has been studied in mice that lack TNF receptor 1 (TNFR1) and TNFR2. The accelerated plaque development in TNFR1-deficient mice suggests that some level of TNFR1 signaling is necessary to protect the arterial wall from atherosclerosis. One must reconcile these contradictory signals that TNF- $\alpha$ may be necessary for both protection and aneurysm growth. Characterization of TNFRmediated signaling events during aneurysm progression in mice may lead to a better understanding of aneurysm formation, growth, and rupture.

\section{Hemodynamics}

The fact that majority of aneurysms occur at arterial bifurcations suggest that hemodynamic stress is a significant factor in IA formation and growth (Gonzalez et al 1992; Hoi et al 2004; Jou et al 2005). Disturbed flow has been a key concept in the onset of thrombosis and inflammation, in part due to recirculating flow in a cavity or at an arterial branch. Blood elements stay in the region for a prolonged period, greatly increasing the adhesion of elements such as platelets and monocytes to endothelial cells (EC). While increasing evidence suggests that high wall stress triggers TNF- $\alpha$ gene expression in ECs that leads to inflammation-mediated dysfunction including expression of MMP in vessel that may degrade the vessel wall, as circulating levels of TNF- $\alpha$ are increased in patients with hemodynamic pressure and volume overload (Kapadia et al 2000).

\section{Hypertension}

Although systemic hypertension is a known risk factor in aneurysm development, it is not understood how systemic hypertension contributes to aneurysm development. It is likely that intravascular pressure subjects the vessel wall to tensile stress, which could cause the aneurysmal sac to enlarge if the focal site of hemodynamic flow is damaged (Kapadia et al 2000). However, this simple view does not uniquely explain how systemic hypertension contributes to the vessel wall degradation that is essential to aneurysm development and growth. We propose that systemic hypertension increases EC calcium and TNF- $\alpha$, which do the initial damage by necrosis of EC providing an opening for vessel out-bulging (Munoz et al 1999). As a result, the aneurysm wall is very nonhomogenous with a disrupted endothelial layer permitting inflammatory macrophages into other compartments of the artery. While hypertension may contribute to the increased level of TNF- $\alpha$, it appears that altered hemodynamic force is a necessary component to promote aneurysm growth as a ready source of inflammatory cells to the vascular wall site.

\section{Gender}

Alterations in vascular function, including reduced bioavailability of nitric oxide and reduction in responses to shear stress with increased cardiovascular risk associated with aging appear related to the onset of menopause (Kongable et al 1996; Henneke and Goldenbock 2001). Female preponderance for $\mathrm{SAH}$ is unclear though it is known that estrogen has an inhibitory effect on aneurysm formation. Since estrogen is known to inhibit TNF- $\alpha$ activity (Srivastava et al 1999; Puder et al 2001; Xing et al 2007), we suggest that reduced estrogen levels in postmenopausal women predispose to a higher incidence of aneurysm development. The collagen content of cerebral arteries may also diminish after menopause, favoring aneurysm formation (Hoh et al 2004). Estrogen attenuates vascular smooth muscle cell (VSMC) expression of adhesion molecules and chemoattractants, and protects against vascular inflammation (Ferreri 2007). The demonstration by Xing and colleagues 
(2007) that estrogen can act as an antagonist of TNF-mediated pro-inflammatory pathways via inhibition of (CINC-2 $\beta)$ is especially noteworthy in this regard (Hoh et al 2004). In agreement with our hypothesis, increased circulating levels of TNF- $\alpha$ have been observed in postmenopausal women (Huang et al 2005; Xing et al 2007). By altering BBB, TNF- $\alpha$ can sustain inflammation by permitting inflammatory molecules (Schurman et al 2001; Ferreri 2007).

\section{Smoke}

Cigarette smoking has been shown, in several studies, to increase the risk for $\mathrm{SAH}$ in all age groups (Longstreth et al 1992; Juvela et al 1993; Weir et al 1998), but the mechanism remains unknown. According to these findings, it appears that smoking increases risk for $\mathrm{SAH}$ by hastening growth of pre-existing aneurysms. Interestingly, the number of cigarettes smoked daily appears to be more important than the duration of smoking, which suggests that the inflammation threshold is significantly increased with the number of cigarettes. It is also interesting to note that cigarette smoking has antiestrogenic properties, indirectly upregulating TNF- $\alpha$ signaling as demonstrated in the pulmonary vessels (Wright et al 2007). While cigeratte smoke can cause many systemic effects (Yanbaeva et al 2007), cigarette smoke could also increase structural fragility by decreasing collagen production (Raveedran et al 2004; Wright et al 2007) and increasing nuclear factor-kappa-B (NFKB) activation (Anto et al 2002).

\section{TNF- $\alpha$ : A key player in inflammation, apoptosis, and arterial degradation}

TNF- $\alpha$ acts via two distinct receptors (Grell et al 1994; Bazzoni and Beutler 1996). TNFR1 is constitutively expressed in most tissues, binds primarily to the soluble form of TNF- $\alpha$ and is the key mediator of TNF- $\alpha$ signaling in many cell types. On the other hand, TNFR2 is typically expressed in endothelial and immune-related cells and is activated by the membrane bound TNF- $\alpha$. Since the affinity of TNF receptor 2 (TNFR2) is five times higher than that for TNFR1 (Pimentel-Muinos and Seed 1999), one dominant effect would be to activate TNF signaling on ECs via NFkB (Figure 1). However, the latter initiates the majority of the biological activities of TNF- $\alpha$. The major difference between the two receptors is the death domain (DD) of TNFR1 that is absent in TNFR2. For this reason, TNFR1 is an important member of the death receptor family that activates both cell death and survival signals (Grell et al 1994; Bazzoni and Beutler 1996; Tang et al 1998; PimentelMuinos and Seed 1999).

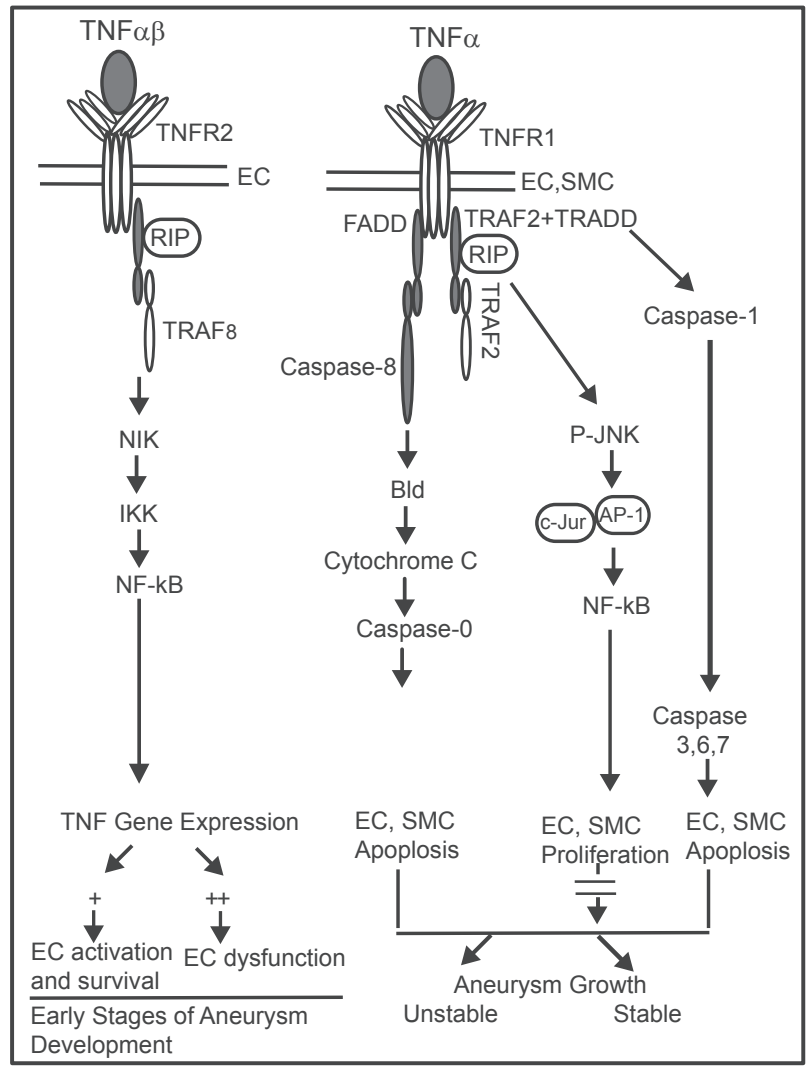

Figure I Proposed TNF- $\alpha$ signaling during aneurysm growth and rupture. TNF- $\alpha$ interaction with TNFRI and TNFR2 elicits either inflammation/survival or apoptosis by recruiting different adaptor proteins. Recruitment of FADD and TRADD leads to activation of apoptosis while recruitment of RIP andTRAF2 generates NFKB, initiating inflammation. During early stages of aneurysm development, membrane bound TNF interacts with TNFR2 and initiates EC activation. The threshold of membrane bound TNF- $\alpha$ appears to control EC activation and survival.While lower TNF- $\alpha$ expression would be expected to activate EC, higherTNF- $\alpha$ concentration would cause EC dysfunction and a fraction of EC death. However, the majority of EC/SMC apoptosis would occur when soluble TNF- $\alpha$ interacts with TNFRI (present on EC and SMC) (Norwak et al 2000). The threshold of soluble TNF- $\alpha$ is critical, as lower concentration would increase SMC proliferation, which might stabilize aneurysms from rupturing (stable aneurysms) (Fig. 4). On the other hand, sustained TNF- $\alpha$ might activate MMPs and other calcium dependent proteases such as calpain promoting aneurysm rupture.

Abbreviations: TNFR, Tumor necrosis factor receptor; FADD, Fas associated death domain;TRADD, TNF receptor associated death domain Receptor; EC, Endothelial cells; SMC, Smooth muscle cells; NFKB, Nuclear factor for kappa B; NIK, NFKB inducing kinase; IKK, Inhibitor of I kappaB kinase; JNK, c-Jun N-terminal kinase; $\mathrm{Bid}, \mathrm{BH} 3$ interacting domain.

The TNF- $\alpha$ signaling via TNFR1 and TNFR2 are depicted in Figure 1 (Grell et al 1994). The TNFR-associated death domain (TRADD) binds to the DD of TNFR1 and recruits the adapter proteins receptor interacting protein (RIP), TNFR-associated factor 2 (TRAF-2), and Fas-associated death domain (FADD). In turn, these adaptor proteins recruit key molecules that are responsible for further intracellular signaling. When TNFR1 signals apoptosis, FADD binds pro-caspase 8 , which is subsequently activated. This activation initiates a protease cascade leading to apoptosis, also involving the mitochondria and with caspases as key regulators (Tantral et al 2004). Alternatively, when TNFR1- 
signals survival, TRAF-2 is recruited to the complex, which inhibits apoptosis via cytoplasmic inhibitor of apoptosis protein (cIAP). The binding of TRAF-2 initiates a pathway of phosphorylation steps resulting in the activation of cFos/cJun transcription factors via mitogen activated protein kinase (MAPK) and cJun N-terminal kinase (cJun). The major signaling event of TRAF-2 and RIP is the widely studied activation of NFKB transcription factor (Pimentel-Muinos and Seed 1999). Both NFkB and cFos/Jun induce transcription of antiapoptotic and pro-inflammatory genes. Given the differential action of TNF- $\alpha$ receptors, we suggest that during early stages of aneurysm formation TNFR2 signaling is activated by membrane-bound TNF- $\alpha$ and when sufficient TNF- $\alpha$ is secreted, it activates TNFR1-signaling resulting inflammation and apoptosis.

TNF- $\alpha$ is known to activate metalloproteinases (MMPs) that increase vascular permeability and hemorrhage (Polavarapu et al 2005). MMPs consist of at least 18 different mammalian zinc- and calcium-dependent proteases, of which monocytes/macrophages express several types. Most MMPs are synthesized in their latent pro-form and converted to their active forms. MMPs have broad substrate specificity in degrading most extracellular proteins and play important roles in normal and pathological conditions. MMP activities are tightly regulated by endogenous inhibitors, (TIMPS), and degrade the principal structural components, collagen and elastin in the artery (Van den Berg et al 1999; Yoneyama et al 2004). Our results indicate reduction in TIMP-1 expression in human ruptured aneurysms with concurrent increase in MMP-9 activity. It is assumed that initiation, growth and rupture are begun from the localized degeneration of the internal elastic membrane at the apices of intracranial arterial bifurcations. This degeneration by the hemodynamically generated forces acts at the apex and weaken the wall (Gonzalez et al 1992). The pulsatile impulse and pressure produces the initial aneurysmal out-pouching. Growth, or enlargement, occurs as a process of structural fatigue due to infiltration of inflammatory cell types and their mediators progressively weaken the structural components of the wall. The architecture of the normal cerebral artery (Figure 2A) with degenerating aneurysm wall is shown to indicate the differences in TNF $\alpha$ signaling (Figure 2B). We propose that aneurysm rupture occurs when the combination of intraaneurysmal pressure, aneurysmal size, and thinning of the aneurysm wall with sustained activation of inflammation by TNF- $\alpha$ exceeds the breaking strength of the structural components of the wall (Figure 2C). We suggest that downregulating TNF- $\alpha$-mediated inflammation (inactive inflammatory cell types) would prevent aneurysm growth and rupture by blocking activation of MMPs (Figure 2D).

\section{TNF- $\alpha$ initiates aneurysm formation through EC dysfunctions}

Though a relatively small organ, the brain is an intricate part of the body that demands and uses $20 \%$ of the volume of blood pumped from the heart. The blood vessels can handle an increased or decreased volume of blood into the brain with an intricate multilayer system of different cell types that work together to support sufficient hemodynamic flow (Ruetzler et al 2001). In normal arteries, the vascular endothelium serves as a natural barrier between the blood and other vascular cell types preventing damage. ECs play a pivotal role in inflammation, in that they constitute the "survey strategy" of the innate immune system to thwart most pathogenic threats. EC activation initiates the inflammatory response by recruiting leukocytes into the damaged arterial site(s); therefore EC dysfunction contributes to the development of a chronic inflammatory response. Leukocyte-endothelial interactions can be divided into three main steps: rolling, firm adhesion, and emigration (Vestweber 2007). The initial tentative contact between circulating white blood cells and the vessel wall, known as rolling, is mediated by the binding of selectin molecules on the endothelium (E-selectin and P-selectin) or leukocytes (L-selectin) to their corresponding ligands. This slows the leukocytes down, essentially increasing the likelihood that they will firmly adhere to the vessel wall via other cell adhesion molecules (CAM), such as intercellular adhesion molecule-1 (ICAM-1) and vascular cell adhesion molecule-1 (VCAM-1) on the vascular endothelium, and integrins such as CD11/18 on the leukocytes. These two families of CAMs are also responsible for the subsequent transmigration of the leukocytes through the vessel wall into the interstitium where they can promote further tissue damage (Figure 2). TNF- $\alpha$ is a potent pro-inflammatory cytokine that triggers strong endothelial activation with increased monocyte adhesion, the hallmark of the inflammatory response. Characterization of TNF-mediated signaling events that cause tissue damage can be therapeutically manipulated using specific antibodies or inhibitors.

While vascular endothelium maintains the integrity of a vessel, vascular smooth muscle cells (VSMC) that are positioned below endothelium play a crucial role in the malleability of the arterial wall. SMC, in concert with the fibrous layers of collagen and elastin, give arteries the plasticity needed to withstand hemodynamic stress without sustaining any significant injury. 


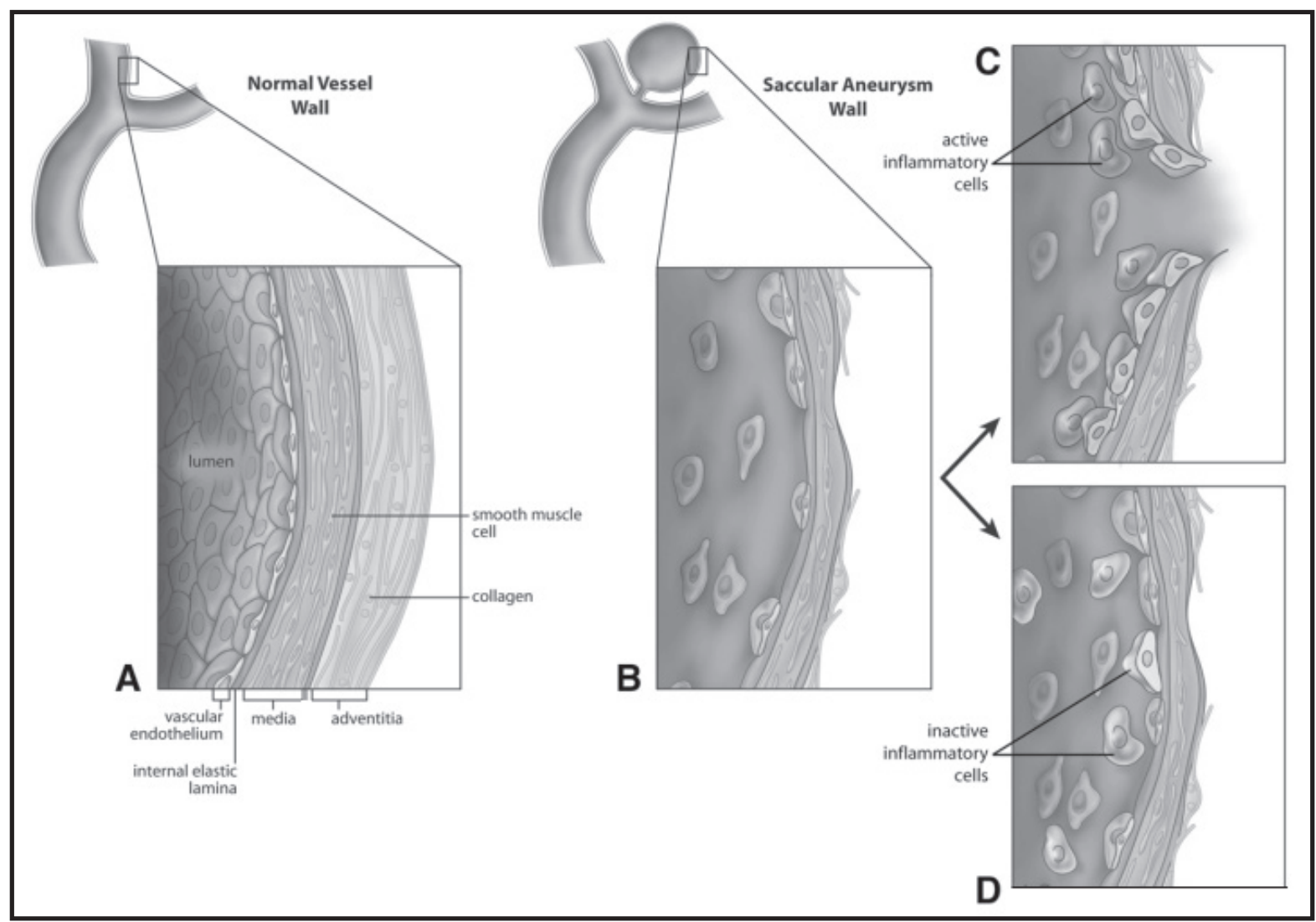

Figure 2 Potential role of TNF- $\alpha$-mediated inflammation in aneurysm progression and rupture. A. Architecture of a normal cerebral artery is shown with endothelial cells at the lumen followed by internal elastic lamina, smooth muscle cells in media, and adventitia. B. Aneurysm wall is characterized by the infiltration of inflammatory cell types that lead to loss of endothelial cells, degeneration of internal elastic lamina and smooth muscle cells. C. Presence of active inflammatory cells (as shown in red) that produce TNF- $\alpha$ is an indicative of aneurysm rupture. D. Inactive inflammatory cells (as shown in green) as a result of either suppressed TNF- $\alpha$ expression or increased anti-inflammatory cytokines are indicative of aneurysm stabilization (nonrupture).

Abbreviation: TNF- $\alpha$, tumor necrosis factor-alpha.

However, this structural integrity is lost in IA and more so in ruptured aneurysms supported by the findings that ruptured aneurysms are more pulsatile than nonruptured aneurysms.

\section{Potential cellular mechanisms that sustain TNF- $\alpha$ expression during IA growth Increased $\mathrm{Ca}^{2+}$-signaling}

Though TNF- $\alpha$ expression is regulated in cells by several factors including nuclear factor for activated $\underline{T}$ (NFAT) activation (an intracellular $\mathrm{Ca}^{2+}$-sensitive transcription factor) and TNF- $\alpha$ converting enzyme (TACE) activity. Studies from several laboratories, including ours, indicate that NFAT activation requires IP3-gated $\mathrm{Ca}^{2+}$-release and $\mathrm{Ca}^{2+}$-influx across the plasma membrane (Jayaraman et al 2000). To determine whether higher TNF- $\alpha$ activity could be due to increased intracellular $\mathrm{Ca}^{2+}$, we performed polymerase chain reaction (PCR) analysis using specific primers for
Inositol 1,4,5-trisphosphate receptor 1 (IP3R1) and IP3R3, predominantly expressed intracellular Ca release channels in inflammatory and endothelial cells. Representative data in the aneurysm wall is shown in Figure 3A, which indicates higher IP3R1 and IP3R3 expression in human ruptured aneurysm wall as compared to control artery (Fig. 3B). The implication of these results could be that up-regulated IP3Rs may increase focal $\mathrm{Ca}^{2+}$ concentration, sustaining $\mathrm{Ca}^{2+}$-dependent signaling molecules activation including TNF- $\alpha$ in human aneurysms.

\section{Toll-like receptors in aneurysm wall}

In response to pathogen invasion, the immune system relies on pathogen sensors before mounting an effective immune response to the pathogen (Aderem and Ulevitch 2000). These pathogen sensors are Toll-like receptors (TLR), which directly bind to the pathogenic ligand leading to an immune response. Most TLR ligands are microbial products that are conserved among a variety of pathogens. TLRs have 
been grouped into families depending on the recognition of ligands (Takeda et al 2003). Lipid-based structures are recognized by TLR2 and bacterial LPS is recognized by TLR4. Viral and bacterial nucleic acids are recognized by TLR3, TLR7, TLR8, and TLR9. Double-stranded RNA (dsRNA) is recognized by TLR3. TLR5 recognizes proteins from pathogens. TLRs seem to require co-receptors for microbial recognition and signaling. For instance CD14, which is present in plasma as a soluble form or on the monocyte cell surface as a glycosylphosphatidylinositol (GPI)-anchored $50 \mathrm{KD}$ protein, binds to LPS and facilitates its signaling. Mice lacking CD14 reveal that CD14 has a role in amplifying the production of TNF- $\alpha$ and IL- 6 during LPS responses (Takada and Aggarwal 2003). Another coreceptor, CD36, mediates homeostatic functions, including adhesion to thrombospondin and collagen and the inhibition of angiogenesis. CD36 acts as a co-receptor for diacyl glyceride (DAG) recognition through the TLR2/6 complex. The fact that expression of several TLRs is increased in our study indicates that bacterial and viral infections in a given individual might leave a component that is recognized by TLR-6 and TLR-2 (Figure 3) (Paul-Clark et al 2006). Given that Gram-positive agonists induce TNF- $\alpha$ in macrophages, it is interesting that Staphylococcus aureus has been noted in bacterial intra-cerebral aneurysms (Byrd-Leiffer et al 2001) and in infected IA (Whitfield et al 1991). We propose that TNF generation and activation by the innate immune system is an important component of the inflammatory response in cerebral arteries because of its ability to alter blood flow and vascular permeability permitting accumulation of immune

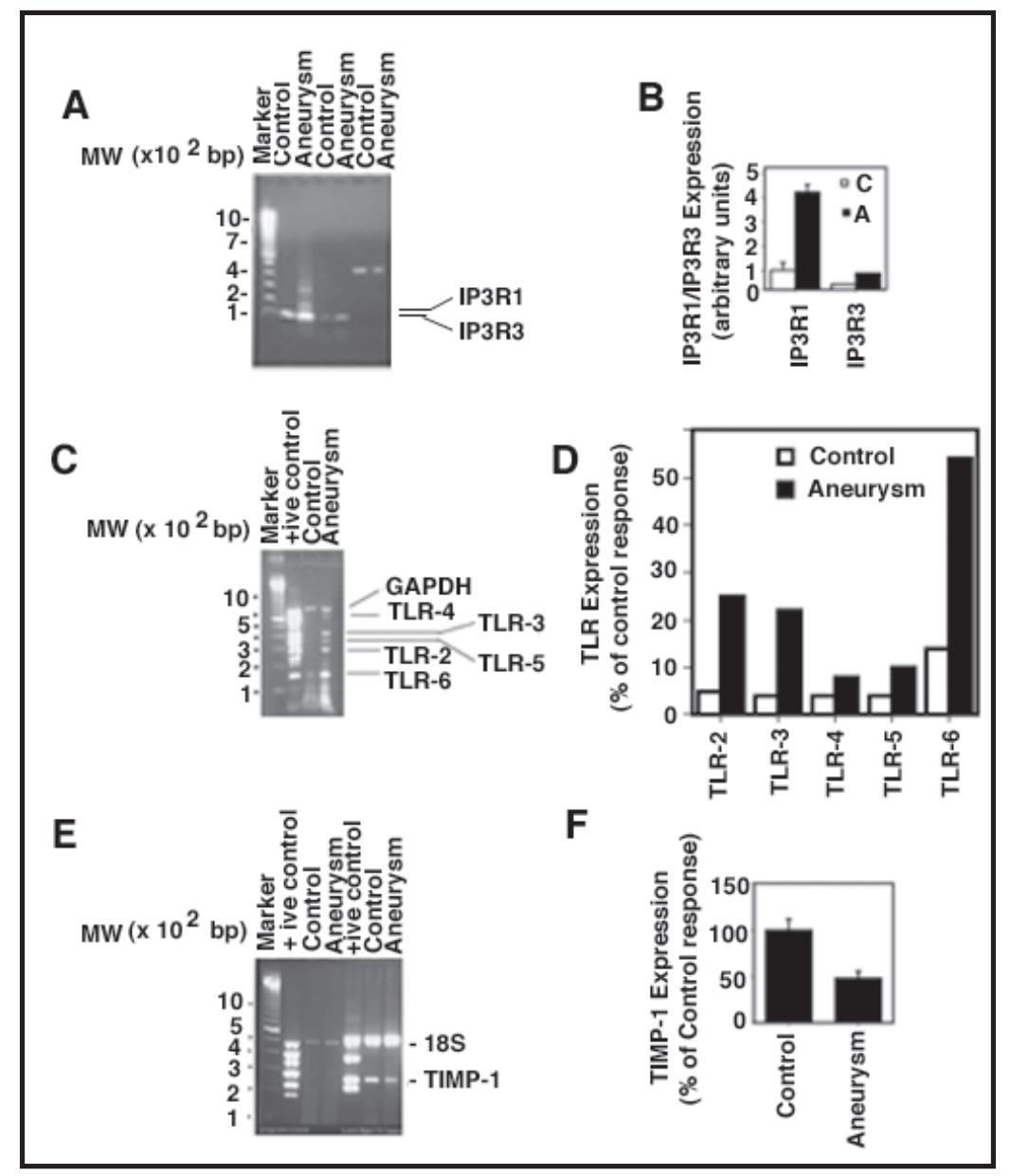

Figure 3 Activation of TNF- $\alpha$ regulatory molecules in human ruptured aneurysms. Representative PCR analysis of IP3RI, IP3R3 (A) and TLRs (C) and TIMP-I (E) using specific oligonucleotide primers. B. Quantitation of IP3RI and IP3R3 (B), TLRs (D), TIMP-I (F) using NIH image software. In all these assays, internal control has been used to normalize quantitation. The lower IP3RI and IP3R3 expression in control artery is not due to insufficient DNA template, as $\beta$-actin expression (a housekeeping gene) was slightly higher in controls than in aneurysm samples (A).

Abbreviations: PCR, Polymerase chain reaction;IP3RI, Inositol I, 4,5-trisphosphate receptor I;TLR,Toll like receptor;TIMP-I,Tissue inhibitor of metalloproteinase- I; GAPDH, Glyceraldehyde-3P-dehydroganase. 
cells in the brain (Karikó et al 2004). Since TLR-2 and TLR-6-mediated TNF- $\alpha$ and IL-6 generation is accompanied by the classical signs of brain-controlled illness responses in rats, it is reasonable to suggest that increased TNF- $\alpha$ expression by these TLRs in aneurysm wall might contribute to the neurological damage (Figure 3C and D) (Hübschle et al 2006). The TLR-2 signaling (as well as TLR-6) is mediated via activation of the myeloid differentiation factor 88 (MyD88) and Myd88 adapter-like (Mal) protein pathways. Detailed analysis of TLRs in nonruptured and ruptured aneurysms would provide a clue on their role in the pathophysiology of aneurysm development.

\section{TIMPI expression is reduced in aneurysm wall}

Extracellular matrix remodeling is associated with and may play a role in the formation and rupture of aneurysms. The aneurysms develop when structural proteins are broken down which allows the arterial wall to bulge out. MMPs are believed to be responsible for damaging the structural proteins and thus contributing to the growth of the aneurysms. The regulator of MMP is a group of TIMP that suppress their activity in normal vessels and their reduction would allow higher MMP activity promoting aneurysm formation. To determine if TIMP expression is reduced in aneurysm walls, we performed PCR analysis using specific primers. Our results show that TIMP-1 expression is significantly reduced in aneurysm walls (Figure $3 \mathrm{E}$ and $\mathrm{F}$ ). Though we have reported that that IP3R, TLR, and TIMP-1 expressions are increased/altered in aneurysm wall, we do not know the induction of these genes is the cause or consequence of these aneurysms growth.

\section{TNF- $\alpha$ threshold is critical for maintaining normal vessel integrity}

Since TNF- $\alpha$ has both protective and detrimental effects on cells under certain conditions, we hypothesize that threshold of TNF- $\alpha$ activity is important in the pathogenesis of cerebral aneurysms growth and rupture (Figure 4). We suggest that during early stages of aneurysm cerebral aneurysm formation, TNF- $\alpha$ expressed is mostly membrane bound on luminal EC, which lead to endothelial activation. However, when EC's express both membrane bound and secrete TNF- $\alpha$, EC dysfunctions and apoptosis occur. Apoptosis of few ECs provides opening for aneurysm formation and for inflammatory cells to infiltrate into inner layers of the vessel to promote aneurysm growth and is facilitated by the hemodynamic flow. TNF- $\alpha$ secretion from inflammatory cells would increase smooth muscle cell (SMC) growth initially (Wang et al 2005), followed by apoptosis when the number of macrophages are increased at the site, which lead to MMP generation that induce structural breakdown. We, therefore, consider TNF- $\alpha$ is a critical signaling molecule involved in berry-type aneurysm pathogenesis. When inflammatory cells are inactivated or reduced below threshold by either anti-inflammatory cytokines or other modulators, SMCs will provide stability to the vessel preventing rupture (nonrupture).

\section{TNF- $\alpha$ polymorphisms in IA}

Fontanella and colleagues (2007) have shown recently that the $\mathrm{G}$ allele, associated with less TNF- $\alpha$ transcription, is more frequent in $\mathrm{SAH}$ and suggest that TNF- $\alpha$ protects aneurysm from rupture. The TNF- $\alpha-308 \mathrm{G} / \mathrm{A}$ polymorphism has been extensively studied in the context of numerous inflammatory

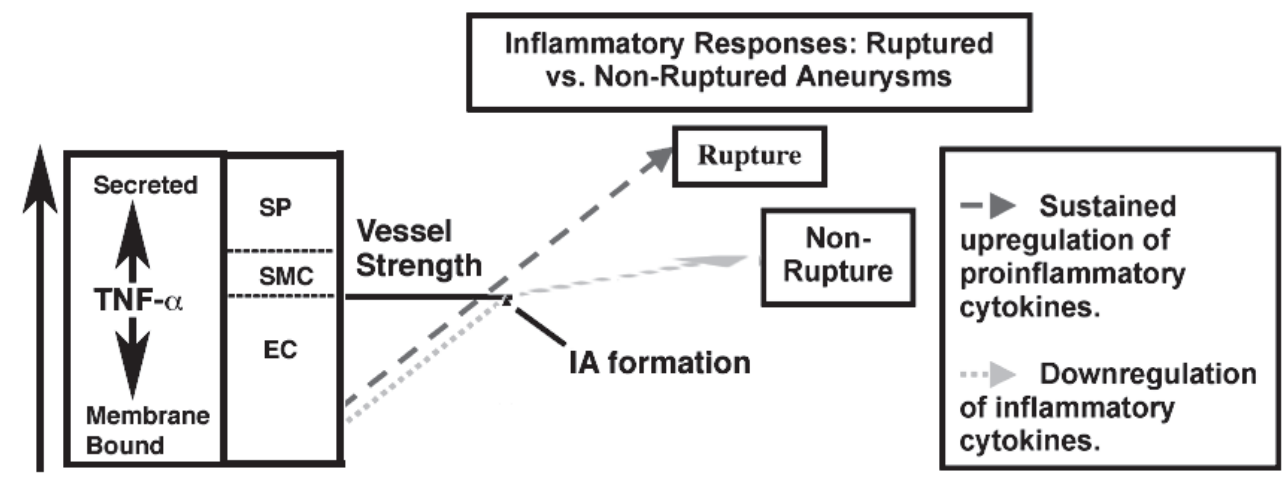

Figure 4 Threshold of TNF $\alpha$ expression is critical for aneurysm growth.TNF- $\alpha$ is a known inducer of inflammation and the extent TNF- $\alpha$ expression correlates the degree of inflammation, which determines aneurysm growth and rupture. SP refers to structural proteins.

Abbreviations: EC, endothelial cell; SMC, smooth muscle cell; SP, structural proteins;TNF- $\alpha$, tumor necrosis factor-alpha. 
disorders. Since inflammatory cell types and reactions are well established in ruptured aneurysms, the differences between our studies cannot be explained. As authors themselves pointed out in this study, additional genetic studies are required in different populations to validate their interesting findings. However, there are several possible explanations. First, it is known that the allele frequencies of single nucleotide polymorphisms (SNPs) differ greatly among different populations/races. Second, while TNF- $\alpha 2$ allele with " $A$ " is a stronger transcriptional activator than the TNF- $\alpha 1$ allele containing " $G$ " (Wilson et al 1992; Hajeer and Hutchinson 2001), there are other SNPs $(-238,-1031,-863$, and -857) that regulate TNF- $\alpha$ as well. For example, TNF- $\alpha$ SNPs, $-1031,-863$, and -857 , variants of which are observed in a relatively large proportion of Japanese, have been suggested to relate to high TNF- $\alpha$ production. In addition, the level of TNF- $\alpha$ production from conconavalin A (ConA) -activated peripheral blood mononuclear cells carrying either $-857 \mathrm{~T}$ or $-863 \mathrm{~A}$ (variant alleles) was significantly higher than those carrying $-857 \mathrm{C}$ and -863C (dominant alleles) (Higuchi et al 1998). Alternatively, additional gene(s), in addition to TNF- $\alpha$, may participate in tandem to influence the individual's susceptibility or severity and may vary in different populations. For example, HLA-DR gene's proximity to TNF- $\alpha$ suggests this possibility (Wilson et al 1993).

Since TNF- $\alpha$ has both protective and detrimental effects on cells under certain conditions, we hypothesize that threshold of TNF- $\alpha$ activity may be important in the pathogenesis of cerebral aneurysms growth and rupture (Figure 4). We suggest that, during early stages of aneurysm cerebral aneurysm formation, TNF- $\alpha$ expressed on luminal EC is mostly membrane-bound, which lead to endothelial activation. However, when EC's express higher levels of TNF- $\alpha$, membrane-bound and secreted forms, which lead to EC dysfunctions and apoptosis, respectively. Apoptosis of few ECs provides opening for aneurysm formation and for inflammatory cells to infiltrate into inner layers of the vessel to promote aneurysm growth and is facilitated by the hemodynamic flow. TNF- $\alpha$ secretion from inflammatory cells would increase SMC numbers initially, followed by apoptosis when the number of macrophages are increased at the site, which lead to MMP generation and structural breakdown. We, therefore, consider TNF- $\alpha$ is a critical signaling molecule involved in berry-type aneurysm pathogenesis. When TNF- $\alpha$ concentration is reduced below threshold by inactivating or reducing inflammatory cells in the vessel wall, SMCs induced by low TNF- $\alpha$ will provide stability to the vessel preventing rupture (stabilized aneurysms). Alternatively,
sTNFRs could also reduce TNF- $\alpha$ activity on vascular cells and stabilize aneurysms.

\section{Therapeutic potential of TNF- $\alpha$}

Though the mechanical integrity of the arterial wall is compromised in the aneurysm wall, the molecular signals from immune cells that initiate aneurysm development and progression are not known. If TNF- $\alpha$ from immune cells is critically important for aneurysm development and growth, it ought to breach the protective barrier of the vascular endothelium by promoting free flow of inflammatory cells mediators over and across endothelium. Many studies have demonstrated the presence of various adhesion molecules and chemokines at sites of inflammation particularly in atherosclerosis. Given enhanced expression of adhesion molecules and chemoattractants in IA, it seems logical to conclude that large numbers of infiltrating leukocytes are recruited into the aneurysm site by these molecules. These chemokines may be anchored to the cell surface, thereby ensuring a relatively high concentration of chemoattractants close to the cerebral aneurysm formation site, hence temporally and spatially restricting activation of circulating cells. It is possible to target and modulate components of the adhesion-migration pathway with TNF- $\alpha$ inhibitors. A complementary strategy would be to down-regulate TNF- $\alpha$ activity in infiltrating leukocytes with intracellular calcium channel inhibitors. Since several inflammatory pathways are dependent on $\mathrm{Ca}^{2+}$ (for instance, TNF- $\alpha$, MMP-9 processing, $\mathrm{NF} \kappa \mathrm{B}$, and NFAT activation), inhibiting $\mathrm{Ca}^{2+}$ concentration may be beneficial.

\section{Future perspectives Use of mouse models to gain insights on human aneurysms}

Since temporal analysis of the genetic changes in humans is not feasible, development of cerebral aneurysms in mice would additionally help to clarify the role of TNF- $\alpha$ in aneurysm growth and rupture. To carefully study the development and growth of aneurysms in vivo, experimental aneurysms at bifurcations of cerebral arteries must 1) recapitulate the essential features of human cerebral aneurysms; and 2) be amenable to experimental manipulation. Transgenic mice or knock out mice can be employed to assess the involvement of specific genes in cerebral aneurysms. Further studies on TNF- $\alpha$ transgenic and null mice will greatly facilitate its role in the patho-physiology of cerebral aneurysms because 1) aneurysm formation and growth can be directly related to the presence and absence of TNF- $\alpha$ expression, respectively; 
2) it would provide better insights on continuous arterial remodeling, as seen in human aneurysms; 3) TNF- $\alpha$ specific antibodies and antagonists as well as soluble TNFR1 and TNFR2 (sTNFR1 and sTNFR2) are easily available to experimentally manipulate and neutralize the TNF- $\alpha$ effects in vivo. Experimental aneurysms in TNF- $\alpha$ transgenic and deficient mice, especially hemodynamically-induced intracranial aneurysms, will be invaluable for establishing a casual relationship among hemodynamic stresses to TNF- $\alpha$ activity changes in inflammation and aneurysm growth. Determining whether TNF- $\alpha$ is involved in the etiology of cerebral aneurysms and hemorrhage may lead to the development of rational therapeutic interventions.

While higher TNF- $\alpha$ expression in aneurysms offers some clues, there are several key questions that remain unanswered: Why do only a few aneurysms rupture? Can TNF- $\alpha$ be involved in both progression and rupture? Is persistent activation a cause of aneurysm rupture? And, is it possible to prevent aneurysm progression and rupture by blocking TNF- $\alpha$ activity? The answers to these questions will lead to a better understanding of aneurysm disease.

\section{Development of small molecule antagonists}

Management of un-ruptured IA is a major health problem due to lack of predictability in terms of growth and rupture (Berenstein et al 1999). As we understand the complexities of inflammation in aneurysm walls, we are presented with several challenges in the quest to develop potent, specific anti-inflammatory agents that do not limit host defense. First, how do we integrate all the information on inflammatory cytokines into our understanding of inflammatory processes? This is required for value to be assigned to the importance of various targets. Second, how useful will it to be to target a major signaling pathway, such as TNF- $\alpha$, which has physiological as well as pathological roles in inflammation? This is a key concern, given the potential problem with targeting TNF- $\alpha$. Inhibitors of this pathway could be useful in the acute context, as in sepsis. However, interfering with specific protein-protein interactions presents attractive possibilities. This receptor interference can be receptor-proximal, or at the level of transcriptional control as specificities of interactions are apparent in the context of aneurysm growth. For instance, differential effects of TNF- $\alpha$-mediated inflammation and apoptosis can be studied by limiting apoptosis and inflammation through antagonists to FADD and $\mathrm{NF} \kappa \mathrm{B}$, respectively.

Finally, developing inhibitors of protein-protein interactions may present a considerable challenge. Even if specific peptide inhibitors with internalization sequences are developed, it seems unlikely that this will form the basis for new drugs. We may have to use the peptides as lead compounds from which to develop peptido-mimetics and/or other compounds. This exciting prospect may prove challenging but the rewards can be tremendous. New treatments to prevent aneurysm growth can be increased by targeting TNF- $\alpha$-mediated inflammation in these patients.

\section{Acknowledgments}

The authors would like to thank the financial support provided by the American Heart Association through Grant-in-Aid and New Investigator Development Award and American Cancer Society (TJ) and the Vascular Biology Fund. I would also like to thank Jill Gregory for her assistance with preparation of the manuscript. There are no conflicts of interest.

\section{References}

Aderem A, Ulevitch RJ. 2000. Toll-like receptors in the induction of innate immune responses. Nature, 406:782-7.

Aggarwal BB, Takada Y. 2005. Cytokines and Cancer. In: Platanias LC (ed). Chicago, IL: Springer, p. 103.

Andrews RJ, Spiegel PK. 1979. Intracranial aneurysms. Age, sex, blood pressure, and multiplicity in an unselected series of patients. $J \mathrm{Neu}$ rosurg, 51:27-32.

Anto RJ, Mukhopadhyay A, Shishodia S, et al. 2002. Cigarette smoke condensate activates nuclear transcription factor-kappa B through phosphorylation and degradation of IkappaB(alpha): correlation with induction of cyclooxygenase-2. Carcinogenesis, 23:1511-18.

Bazzoni F, Beutler B. 1996. Tumor necrosis factor ligand and receptor family. N Engl J Med, 334:1717-23.

Benelli R, Lorusso G, Albini A, et al. 2006. Cytokines and chemokines as regulators of angiogenesis in health and disease. Curr Pharm Des, $12: 3101-15$.

Berenstein A, Flamm ES, Kupersmith MJ. 1999. Unruptured intracranial aneurysms. $N$ Engl J Med, 340:1439-40.

Byrd-Leifer CA, Block EF, Takeda K, et al. 2001. The role of MyD88 and TLR4 in the LPS-mimetic activity of taxol. Eur J Immunol, 31:2448-57.

Cao Y, Zhao J, Wang S, et al. 2002. Monocyte chemoattractant protein-1 mRNA in human intracranial aneurysm walls. Zhonghua Yu Fang Yi Xue Za Zhi, 36:519-21.

Chen ZK, Lv HS. 2006. Quantification of expression of leukotriene B4 inducing tumor necrosis factor-alpha and interleukin-1beta at mRNA level in synovial membrane cells of rheumatoid arthritis by real-time quantitative PCR. Beijing Da Xue Xue Bao, 38:533-6.

Chyatte D, Bruno G, Desai S, et al. 1999. Inflammation and intracrania aneurysms. Neurosurgery, 45:1137-55.

Ferreri NR. 2007. Estrogen-TNF interactions and vascular inflammation. Am J Physiol Heart Circ Physiol, 292:H2566-9.

Fontanella M, Rainero I, Gallone S, et al. 2007. Tumor necrosis factor-alpha gene and cerebral aneurysms. Neurosurg, 60:668-72.

Gonzalez CF, Cho YI, Ortega HV, et al. 1992. Intracranial aneurysms: flow analysis of their origin and progression. AJNR Am J Neuroradiol, 13:181-8.

Gregersen R, Lambertsen K, Finsen B. 2000. Microglia and macrophages are the major source of tumor necrosis factor in permanent middle cerebra artery occlusion in mice. J Cereb Blood Flow Metab, 20:53-65.

Grell M, Zimmermann G, Hulser D, et al. 1994. TNF receptors TR60 and TR80 can mediate apoptosis via induction of distinct signaling pathways. J Immunol, 153:1963-72. 
Hajeer AH, Hutchinson IV. 2001. Influence of TNF alpha gene polymorphisms on TNF alpha production and disease. Hum Immunol, 62:1191-9.

Härtel C, Adam N, Strunk T, et al. 2005. Cytokine responses correlate differentially with age in infancy and early childhood. Clin Exр Iттиnol, 142:446-53.

Hashimoto T, Meng H, Young WL. 2006. Intracranial aneurysms: links among inflammation, hemodynamics and vascular remodeling. Neurol Res, 28:372-80.

Henneke P, Goldenbock DT. 2002. Innate immune recognition of lipopolysaccharide by endothelial cells. Crit Care Med, 30:207-13.

Henry PD, Chen CH. 1993. Inflammatory mechanisms of atheroma formation: Influence of fluid mechanics and lipid-derived inflammatory mediators. Am J Hypertens, 6:328S-34S.

Higuchi T, Seki N, Kamizono S, et al. 1998. Polymorphism of the 5 '-flanking region of the human necrosis factor (TNF) - alpha gene in Japanese. Tissue Antigens, 51:605-12.

Hoi Y, Meng H, Woodward SH, et al. 2004. Effects of arterial geometry on aneurysm growth: three-dimensional computational fluid dynamics study. J Neurosurg, 101:676-81.

Hotamisligil GS. 2006. Inflammation and metabolic disorders. Nature, 444:860-7.

Huang Y, Cao S, Nagamani M, et al. 2005. Decreased circulating levels of tumor necrosis factor-alpha in postmenopausal women during consumption of soy containing isoflavones. J Clin Endocrinol Metab, 90:3956-62.

Hübschle T, Mütze J, Mühlradt PF, et al. 2006. Pyrexia, anorexia, adiposia, and depressed motor activity in rats during systemic inflammation induced by the Toll-like receptors- 2 and -6 agonists MALP-2 and FSL-1. Am J Physiol Regul Integr Comp Physiol, 290:R180-7.

Jang IK, Lassila R, Fuster V. 1993. Atherogenesis and inflammation. Eur J Heart, 14(Suppl K):2-6.

Jayaraman T, Berenstein V, Li X, et al. 2005. Tumor necrosis factor alpha in cerebral aneurysms: A key modulator in inflammation. Neurosurgery, 57:558-64.

Jou LD, Wong G, Dispensa B, et al. 2005. Correlation between luminal geometry changes and hemodynamics in fusiform intracranial aneurysms. AJNR Am J Neuroradiol, 26:2357-63.

Juvela S, Hillbom M, Numminen H, et al. 1993. Cigarette smoking and alcohol consumption as risk factors for aneurismal subarachnoid hemorrhage. Stroke, 24:639-46.

Juvela S, Porras M, Poussa K. 2000. Natural history of unruptured intracranial aneurysms: probability and risk factors for aneurysm rupture. Neurosurg Focus, 93:379-87.

Juvela S. 2002. Natural history of unruptured intracranial aneurysms: risks for aneurysm formation, growth, and rupture. Acta Neurochir Suppl, 82:27-30.

Kapadia SR, Yakoob K, Nader S, et al. 2000. Elevated circulating levels of serum tumor necrosis factor-alpha in patients with hemodynamically significant pressure and volume overload. J Am Coll Cardiol, 36:208-12.

Karikó K, Weissman D, Welsh FA. 2004. Inhibition of toll-like receptor and cytokine signaling - a unifying theme in ischemic tolerance. $J$ Cereb Blood Flow Metab, 24:1288-304.

Kataoka K, Taneda M, Asai T, et al. 1999. Structural fragility and inflammatory response of ruptured cerebral aneurysms. A comparative study between ruptured and unruptured cerebral aneurysms. Stroke, 30:1396-401.

Kirwan JP, Krishnan RK, Weaver JA, et al. 2001. Human aging is associated with altered TNF-alpha production during hyperglycemia and hyperinsulinemia. Am J Physiol Endocrinol Metab, 281:E1137-43.

Kongable GL, Lanzino G, Germanson TP, et al. 1996. Gender-related differences in aneurysmal subarachnoid hemorrhage. J Neurosurg, 84:43-8.

Krex D, Rohl H, Koing IR, et al. 2003. Tissue inhibitor of metalloproteinases-1, -2 , and -3 polymorphisms in a white population with intracranial aneurysms. Stroke, 34:2817-21.

Krings T, Piske RL, Lasjaunias PL. 2005. Intracranial arterial aneurysm vasculopathies: targeting the outer vessel wall, 47:931-7.
Kuldo JM, Westra J, Asgeirsdottir SA, et al. 2005. Differential roles of NFKB inhibitors and combinations thereof on TNF- $\alpha$ and IL- $1 \beta$ induced pro-inflammatory status of endothelial cells in vitro. Am J Physiol Cell Physiol, 89:C1229-39.

Laflamme N, Rivest S. 2001. Toll-like receptor 4: the missing link of the cerebral innate immune response triggered by circulating gram-negative bacterial cell wall components. FASEB $J, 15: 155-63$.

Lambertsen KL, Gregersen R, Finsen B. 2002. Microglial-macrophage synthesis of tumor necrosis factor after focal cerebral ischemia in mice is strain dependent. J Cereb Blood Flow Metab, 22:785-97.

Lanzke N, Kleinwächter R, Kerschischnik S, et al. 2007. Differential effects of ethanol on IFN-gamma- and TNF-alpha-producing splenic T lymphocytes in a murine model of Gram-negative pneumonia. Addict Biol, 12:59-68.

Longstreth WT Jr, Nelson LM, Koepsell TD, et al. 1992. Cigarette smoking, alcohol use, and subarachnoid hemorrhage. Stroke, 23:1242-9.

Luedemann C, Bord E, Qin G, et al. 2005. Ethanol modulation of TNF-alpha biosynthesis and signaling in endothelial cells: synergistic augmentation of TNF-alpha mediated endothelial cell dysfunctions by chronic ethanol. Alcohol Clin Exp Res, 29:930-8.

Magder S, Neculcea J, Neculcea V, et al. 2006. Lipopolysaccharide and TNF-alpha produce very similar changes in gene expression in human endothelial cells. $J$ Vasc Res, 43:447-61.

Maini RN, Elliott MJ, Brennan FM, et al. 1995. Monoclonal anti-TNF alpha antibody as a probe of pathogenesis and therapy of rheumatoid disease. Immunol Rev, 144:195-223.

Meng H, Heng Y, Woodward SH, et al. 2005. Mathematical model of the rupture mechanism of intracranial saccular aneurysms through daughter aneurysm formation and growth. Neurol Res, 27:459-65.

Morgan L, Hawe E, Palmen J, et al. 2005. Polymorphism of the heme oxygenase-1 gene and cerebral aneurysms. Br J Neurosurg, 19:317-21.

Morgan L, Cooper J, Montgomery H, et al. 2006. The interleukin-6 gene$174 \mathrm{G}>\mathrm{C}$ promoter polymorphisms are related to cerebral aneurysms. J Neurol Neurosurg Psychiatry, 77:915-17.

Moriwaki T, Takagi Y, Sadamasa N, et al. 2006. Impaired progression of cerebral aneurysms in interleukin-1 beta deficient mice. Stroke, 37:900-5.

Mosca L, Appel LJ, Benjamin EJ, et al. 2004. Evidence based guidelines for cardiovascular disease prevention in women. Circulation, 109:672-93.

Muñoz J, Albillos A, Pérez-Páramo M, et al. 1999. Factors mediating the hemodynamic effects of tumor necrosis factor-alpha in portal hypertensive rats. Am J Physiol, 276:G687-93.

Nagel T, Resnick N, Dewey CF Jr, et al. 1999. Vascular endothelial cells respond to spatial gradients in fluid shear stress by enhanced activation of transcription factors. Arterioscler Thromb Vasc Biol, 19:1825-34.

Nahed BV, Bydon M, Ozturk AK, et al. 2007. Genetics of intracranial aneurysms. Neurosurgery, 60:213-25.

Norwak M, Gaines GC, Rosenberg J, et al. 2000. LPS-induced liver injury in D-galactosamine-sensitized requires secretion of TNF-alpha and the TNF-p55 receptor. Am J Physiol Regul Intergr Comp Physiol, 278: R1202-9.

Ostergaard JR. 1989. Risk factors in intracranial aneurysms: aspects on the formation and rupture of aneurysms, and development of cerebral vasospasm. Acta Neurol Scand, 80:81-98.

Paolisso G, Rizzo MR, Mazziotti G, et al. 1998. Advancing age and insulin resistance: role of plasma tumor necrosis factor-alpha. Am J Physiol, 275:E294-9.

Parker CW, Aykent S. 1982. Calcium stimulation of the 5-lipoxygenase from RBL-1 cells. Biochem Biophys Res Comm, 109:1011-16.

Paul-Clark M, McMaster SK, Belcher E, et al. 2006. Differential effects of gram positive versus gram negative bacteria on NOSII and TNF $\alpha$ in macrophages: role of TLR's in synergy between the two. Br J Pharmacol, 148:1067-75

Pavic M, Debourdeau P, Teixeira L, et al. 2001. Bacterial cerebral aneurysms without infectious endocarditis: analysis of a case and review of the literature. Rev Med Interne, 22:867-71. 
Peschon JJ, Torrance DS, Stocking KL, et al. 1998. TNF deficient mice reveal divergent roles for $\mathrm{p} 55$ and $\mathrm{p} 75$ in several models of inflammation. J Immunol, 160:943-52.

Peters DG, Kassam AB, Feingold E, et al. 2001. Molecular anatomy of an intracranial aneurysm coordinated expression of genes involves in wound healing and tissue remodeling. Stroke, 32:1036-42.

Pimentel-Muinos FX, Seed B. 1999. Regulated commitment of TNF receptor signaling: a molecular switch for death or activation. Immunity, 11:783-93.

Polavarapu R, Gongora MC, Winkles JA, et al. 2005. Tumor necrosis factor-like weak inducer of apoptosis increases the permeability of the neurovascular unit through nuclear factor-kappa B pathway activation. $J$ Neurosci, 25:10094-100.

Puder JJ, Freda PU, Goland RS, et al. 2001. Estrogen modulates the hypothalamic-pituitary-adrenal and inflammatory cytokine responses to endotoxin in women. $J$ Clin Endocrinol Metab, 86:2403-8.

Raveedran M, Senthil D, Utama B, et al. 2004. Cigarette suppresses the expression of $\mathrm{P} 4 \mathrm{H} \alpha$ and vascular collagen production. Biochem Biophys Res Comm, 323:592-8.

Ruetzler CA, Furuya K, Takeda H, et al. 2001. Brain vessels normally undergo cyclic activation and inactivation: evidence from tumor necrosis factor-alpha, heme oxygenase-1, and manganese superoxide dismutase immunostaining of vessels and perivascular brain cells. $J$ Cereb Blood Flow Metab, 21:244-52.

Ruigrok YM, Rinkel GJE, Wimjenga C, 2005. Genetics of intracranial aneurysms. Lancet Neurol, 4:179-89.

Schurman L, Sedlinsky C, Mangano A, et al. 2001. Estrogenic status influences nitric oxide-regulated TNF-alpha release from human peripheral blood monocytes. Exp Clin Endocrinol Diabetes, 109:340-4.

Sirén AL, McCarron R, Wang L, et al. 2001. Proinflammatory cytokine expression contributes to brain injury provoked by chronic monocyte activation. Mol Med, 7:219-29.

Slifka MK, Whitton JL. 2000. Clinical implications of dysregulated cytokine production. $J \mathrm{Mol} \mathrm{Med,} \mathrm{78:74-80.}$

Srivastava S, Weitzmann MN, Cenci S, et al. 1999. Estrogen decreases TNF $\alpha$ gene expression by blocking JNK activity and the resulting production of c-Jun and JunD. J Clin Invest, 104:503-13.

Sun SH, Tang WX, Liu C, et al. 2007. Effects of tumor necrosis factor alpha on proteolysis of respiratory muscles in rats with chronic obstructive pulmonary disease. Zhonghua Jie He He Hu Za Zhi, 30:186-91.

Takada Y, Aggarwal BB. 2003. Genetic deletion of the tumor necrosis factor receptor p60 or p 80 sensitizes macrophages to lipopolysaccharideinduced nuclear factor-kappa B, mitogen-activated protein kinases, and apoptosis. J Biol Chem, 278:23390-7.

Takeda K, Akira S. 2003. Toll receptors and pathogen resistance. Cell Microbiol, 5:143-53.

Takeuchi O, Hoshino K, Kawai T, et al. 1999. Differential roles of TLR2 and TLR4 in recognition of gram-positive and gram-negative bacterial cell wall components. Immunity, 11:443-51.

Tang V, Dhirapong A, Yabes AP, et al. 2005. TNF- $\alpha$-mediated apoptosis in vascular smooth muscle cells requires p73. Am J Physiol Cell Physiol, 289:199-206.
Toder RD, Lewis I, Bruno G, et al. 1998. Identification of a serum gelatinase associated with the occurrence of cerebral aneurysms as pro-matrix metalloproteinase-2. Stroke, 29:1580-3.

Tracy KJ, Cerami A. 1993. Tumor necrosis factor, other cytokines and disease. Ann Rev Cell Biol, 9:317-43.

Van den Berg JSP, Pals G, Arwert F, et al. 1999. Type III collagen deficiency in saccular intracranial aneurysms: defect in gene regulation? Stroke, 30:1628-31.

Vestweber D. 2007. Adhesion and signaling molecules controlling the transmigration of leukocytes through endothelium. Immunol Rev, 218:178-96.

Wang Z, Rao PJ, Castresana MR, et al. 2005. TNF-alpha induces proliferation or apoptosis in human saphenous vein smooth muscle cells depending on phenotype. Am J Physiol Heart Circ Physiol, 288: H293-301.

Weir BK, Kongable GL, Kassell NF, et al. 1998. Cigarette smoking as a cause of aneurismal subarachnoid hemorrhage and risk for vasospasm: a report of the Cooperative Aneurysm Study. J Neurosurgery, 89:405-11.

Weiss T, Grell G, Siemienski K, et al. 1998. TNFR80-dependent enhancement of TNFR60-induced cell death is mediated by TNFR-associated factor 2 and is specific for TNFR60. J Immunol, 161:3136-42.

Whitfield PC, Bullock R. 1991. Infected intracranial aneurysm in an infant: case report. Neurosurgery, 28:623-5.

Wilson AG, di Giovine FS, Blakemore AI, et al. 1992. Effects of a polymorphism in the human tumor necrosis factor alpha promoter on transcriptional activation. Proc Natl Acad Sci USA, 94:3195-9.

Wilson AG, deVries N, Pociot F, et al. 1993. An allelic polymorphism within the human tumor necrosis factor alpha promoter region is strongly associated with HLA A1, B8 and DR3 alleles. $J$ Exp Med, 177:557-60.

Wright JL, Tai H, Wang R, et al. 2007. Cigarette smoke upregulates vascular matrix metalloproteinases via TNF-alpha signaling. Am J Physiol Lung Cell Mol Physiol, 292:L125-33.

Xing D, Feng W, Miller AP, et al. 2007. Estrogen modulates TNF- $\alpha$ induced inflammatory responses in rat aortic smooth muscle cells through estrogen receptor- $\beta$ activation. Am J Physiol Heart Circ Physiol, 292: H2607-12.

Xing Z, Gauldie J, Cox G, et al. 1998. IL-6 an anti-inflammatory cytokine required for controlling local or systemic acute inflammatory responses. J Clin Invest, 101:311-20.

Yanbaeva DG, Dentener MA, Creutzberg EC, et al. 2007. Systemic effects of smoking. Chest, 131:1557-66.

Yoneyama T, Kasuya H, Onda H, et al. 2004. Collagen type $1 \alpha 2$ (Col1A2) is the susceptible gene for intracranial aneurysms. Stroke, 35:443-8.

Zhang B, Dhillon S, Geary I, et al. 2001. Polymorphisms in matrix metalloproteinase-1, $-3,-9$, and -12 in relation to subarachnoid hemorrhage. Stroke, 32:2198-202.

Zhao L, Moos MP, Grabner R et al. 2004. The 5-lipoxygenase pathways promote pathogenesis of hyperlipidemia-dependent aortic aneurysm. Nat Med, 10:966-73. 
